\title{
Effect of saliency and L1-L2 similarity on the processing of English past tense by French learners: an ERP study
}

\author{
Maud Pélissier ${ }^{1}$, Jennifer Krzonowski ${ }^{2}$, Emmanuel Ferragne ${ }^{1}$ \\ ${ }^{1}$ Laboratoire CLILLAC-ARP, EA 3967, Université Paris Diderot, France \\ ${ }^{2}$ Laboratoire DDL, UMR 5596, CNRS - Université Lyon 2, France \\ https://doi.org/10.36505/ExLing-2016/07/0031/000290
}

\begin{abstract}
This study explored the effect of saliency and L1-L2 similarity on the processing of second language morphosyntax. ERP responses to violations of past tense morphology were obtained from adult intermediate French learners of English. Results show that participants processed L2-specific violations as salient events and not as morphosyntactic incongruities.
\end{abstract}

Key words: ERPs, L2 processing, syntax, L1-L2 similarity, saliency

\section{Introduction}

The way the syntax of our first language (L1) interacts with the syntax of a language we are trying to learn (L2) remains a much debated issue in the field of SLA. Some of the possible facilitating factors include the presence of similar structures in the L1 and the saliency of the morphosyntactic structure under scrutiny in the L2 (MacWhinney, 2005). In this study, we focused on a structure that contrasts these two factors: ERP responses to morphosyntactic violations of the past tense in polar questions in French learners of English with the auxiliaries DID and HAD. Polar questions using HAD followed by a past participle work in a way that is similar to French, where the past tense is marked both on the auxiliary and the main verb. On the contrary, questions with DID are specific to English in that the past tense is marked only on the auxiliary. However, violations of past-tense inflection are phonologically more salient with DID, where a past morpheme is added to the main verb, than with HAD.

\section{Methods \\ Participants}

26 intermediate French learners of English (5 male, aged $18.5 \pm 1$ ) took part in the experiment. They were first year University students of English having spent less than a month in an English-speaking country.

ExLing 2016: Proceedings of $7^{\text {th }}$ Tutorial and Research Workshop on Experimental Linguistics, 27 June - 2 July 2016, Saint Petersburg, Russia 


\section{Materials and Procedure}

The material consisted of 192 simple polar questions, half of them containing the auxiliary DID (DID Condition) and half HAD (HAD condition). Half of the sentences in each condition were made incorrect by varying the presence of the past morpheme. 120 sentences containing other agreement violations and 120 sentences containing a semantic violation were added as fillers.

Participants were asked to focus on the meaning of the sentence and evaluate its semantic acceptability while EEG data were recorded. A fixation cross appeared first for $500 \mathrm{~ms}$ and remained on the screen during the auditory presentation of the stimulus and for $1000 \mathrm{~ms}$ afterwards. A screen then prompted the participant to evaluate the semantic acceptability of the sentence by pressing a coloured button. As soon as the participant answered or after $2000 \mathrm{~ms}$, the fixation cross appeared again and the next stimulus was presented.

Participants also completed a timed Grammaticality Judgment Task (GJT) with similar stimuli and additional fillers.

\section{EEG data acquisition and analysis}

EEGs were recorded with a Biosemi Active'Two system with 32 active electrodes, referenced on-line to the two mastoids and re-referenced offline to the average of the two mastoids. Data were filtered on-line between 0.1 and $100 \mathrm{~Hz}$. Electrode impedance was maintained below 20 Ohms and the signal was sampled at a rate of $512 \mathrm{~Hz}$. Epochs from -200 $\mathrm{ms}$ to $1000 \mathrm{~ms}$ around the critical point (beginning of the critical past morpheme) were extracted from continuous data. After baseline correction (-200-0 ms) and low-pass filtering at $30 \mathrm{~Hz}$, trials for which peak-to-peak amplitude exceeded $70 \mu \mathrm{V}$ on the EOG channel or $100 \mu \mathrm{V}$ on the other channels were automatically rejected. Electrodes were divided into central and lateral sites, the latter also divided into anterior/posterior region and left/right hemisphere. The following temporal windows were selected: $600-900 \mathrm{~ms}$ for the P600 and 300-500 ms for the LAN or N400.

\section{Results}

\section{Behavioural measures: the GJT}

A sensitivity index (d) was computed for each participant and each auxiliary. Analyses showed that the participants' $d$ ' was marginally better in the Had condition $(F(1,25)=3.48, p=.07)$ but their response time was shorter with DID $(F(1,25)=7.98, p<.01)$ : on average, it took them 562 $\mathrm{ms}$ to respond to sentences containing DID and $634 \mathrm{~ms}$ for sentences containing HAD. 


\section{EEG results}

A repeated-measures ANOVA with mean amplitude in the P600 window as dependent variable and Condition (Correct / Incorrect), Auxiliary (DID / HAD), Hemisphere (Left / Right) and Region (Anterior / Posterior) as within-subject variables showed an effect of the interaction between Condition and Auxiliary $(F(1,28)=9.15, p<.01)$. Post-hoc analyses revealed that the effect of Condition in this time window was limited to sentences with DID $(p<.001)$. A similar ANOVA was conducted on the mean amplitude in the 300-500 ms window and an effect of the Condition $\times$ Auxiliary interaction $(F(1,28)=25.68, p<.001)$ was found. Post-hoc analyses revealed that with DID, the amplitude was greater in the Incorrect than in the Correct condition $(p<.001)$ but that with HAD, the amplitude was more negative in the Incorrect than in the Correct Condition $(p<.001)$.

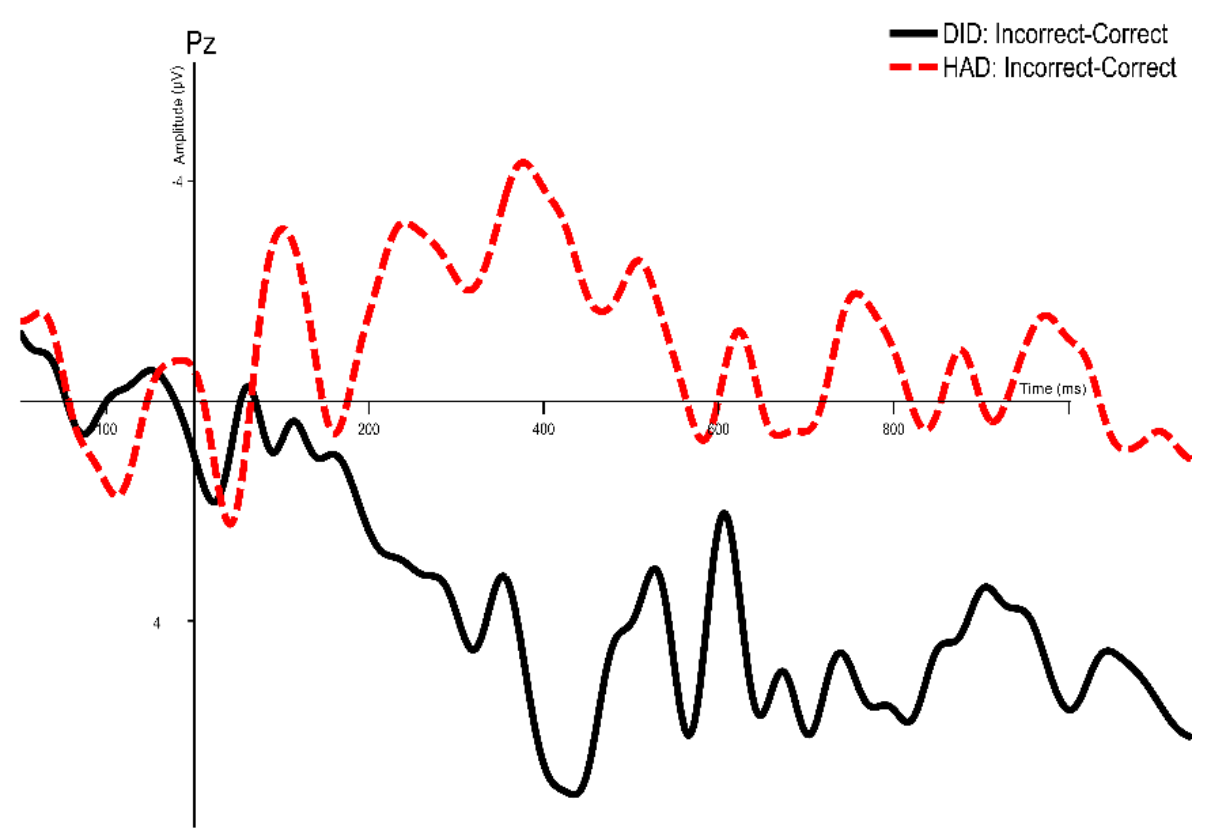

Figure 1. Difference wave (Incorrect - Correct) for each Auxiliary at Pz.

\section{Discussion}

Violations in the DID condition thus elicited a P600 as well as a positive peak in the $300-500 \mathrm{~ms}$ window, resembling a P3 component. These violations involve the presence of the past morpheme in a context where it should be absent. They are therefore more phonetically salient than violations with $\mathrm{HAD}$, which are due to the absence of this same 
morpheme. These results are therefore consistent with the hypothesis that the P600 reflects, as the P3 does, the subjective salience of the stimulus (Sassenhagen, Schlesewsky, \& Bornkessel-Schlesewsky, 2014). Besides, polar questions with DID represent a complex L2-specific structure, since they involve the movement of the inflectional morpheme from the main verb (where it would be in a declarative sentence) to the auxiliary. This represents an additional processing cost; yet participants were faster to decide for these sentences. This apparent discrepancy, as well as the presence of the P3, suggests that the P600 effect observed here in the DID condition is not a reflection of a better perception of the morphosyntactic error at hand but of an explicit reaction to the superior saliency of this violation.

Violations in the HAD condition elicited a negativity in the 300$500 \mathrm{~ms}$ window that was not limited to anterior sites, thus more reminiscent of an N400 than a LAN. N400 effects have been found to be elicited by morphosyntactic violations even in native speakers (Tanner \& Van Hell, 2014), possibly because those speakers rely more on lexicosemantic information to process their native language. It thus seems that these violations with $\mathrm{HAD}$ were not perceived as subjectively salient events but as lexical violations.

These results suggest that when the processed structure does not exist in the L1, other cues such as the phonological salience of the violation are used to process morphosyntactic violations. These findings also have theoretical relevance since they strongly support the P600-asP3 hypothesis.

\section{Acknowledgements}

This research was supported by an IUF grant awarded to Dr. Emmanuel Ferragne.

\section{References}

MacWhinney, B. 2005. Extending the Competition Model. International Journal of Bilingualism, 9(1), 69-84.

Sassenhagen, J., Schlesewsky, M., \& Bornkessel-Schlesewsky, I. 2014. The P600-as-P3 hypothesis revisited: Single-trial analyses reveal that the late EEG positivity following linguistically deviant material is reaction time aligned. Brain and Language, 137, 29-39.

Tanner, D., \& Van Hell, J. G. 2014. ERPs reveal individual differences in morphosyntactic processing. Neuropsychologia, 56(1), 289-301. 